\title{
Empyema necessitatis secondary to Staphylococcus aureus lung abscess
}

\author{
Hawa Edriss MD, Gilbert Berdine MD
}

\begin{abstract}
A 60-year-old man with a past medical history of tobacco abuse, remote alcohol abuse, and left total hip arthroplasty presented with a left upper chest wall mass and left hip and leg pain. Six days prior to presentation, the patient tripped and fell on his back on a hardwood floor. On the following day, he started having left shoulder, left chest wall, and left leg pain. He visited the local emergency department (ED) and was discharged home on ibuprofen. The left leg pain improved, but the chest wall pain got worse, and he noted a rounded painful swelling of the left upper chest. The patient returned to his local ED and was transferred to our facility for further evaluation. He reported chills, high grade fever $\left(104^{\circ} \mathrm{F}\right)$, and productive cough for the past six days with yellow-orange sputum. The patient denied weight loss, hemoptysis, and night sweats. He has a greater than 30 pack-year smoking history, smokes marijuana, and drinks one beer per week. He used to work as a painter.
\end{abstract}

His physical examination revealed a soft partially compressible round subcutaneous mass $(4 \times 7 \mathrm{~cm})$ in the left chest wall just below the clavicle. His vital signs included temperature $101.5^{\circ} \mathrm{F}$, blood pressure $152 / 69 \mathrm{mmHg}$, heart rate 109 beats/minute, respiratory rate 26 breaths/ minute, and oxygen saturation $94 \%$ on room air. Laboratory results revealed a white blood count of $22.5 \mathrm{k} / \mu \mathrm{L}$. Chest $\mathrm{x}$-ray showed an ill-defined $3 \times 4 \mathrm{~cm}$ cavity in the left upper lobe (LUL). Computed tomography (CT) of the thorax demonstrated a thick walled cavity measuring $3.3 \times 3.9 \mathrm{~cm}$ in size in the LUL. Multiple gas locules extending from the cavity into the anterior chest wall along the left sternoclavicular joint $(4.5 \times 3.9 \mathrm{~cm})$, deep to the left pectoralis muscles $(2.3 \times 4.1 \mathrm{~cm})$, and deep to the sternum in the anterior mediastinum $(1.8 \times 5.5 \mathrm{~cm}$ in

Corresponding author: Hawa Edriss

Contact Information: Hawa.Edriss@ttuhsc.edu DOI: $10.12746 /$ swrcc. v5i20.413 size) (Figure) were present. The mediastinal and left hilar lymph nodes were slightly enlarged, and there were bilateral pleural effusions.

The patient was started on vancomycin and meropenem. Sputum examination demonstrated sheets of acute inflammatory cells admixed with macrophages and squamous cells, consistent with an infectious/inflammatory process. Methicillin resistant Staphylococcus aureus (MRSA) was isolated from his sputum culture. His blood cultures were negative. The patient underwent a wedge resection of the left upper lobe. Histopathological examination revealed organizing pneumonia; alveolar spaces were filled with fibrous plugs or acute inflammatory exudates, and the pleura was markedly thickened with lymphocytic infiltrates (pachypleuritis). No neoplasm or granulomatous inflammation was noted. No fungi were seen with the GMS stain. During this hospitalization, the patient was also treated for methicillin-sensitive Staphylococcus aureus (MSSA) left hip septic arthropathy, and he underwent total hip arthroplasty hardware removal with antibiotics spacer implantation. He was discharged home in good condition to complete a total of 6-8 weeks of intravenous antibiotics.

\section{Discussion}

Empyema necessitatis is a rare complication of lung abscess and empyema due to rupture of pus in the pleural space into adjacent tissue. Although it is unclear why, the anterior chest wall between the anterior axillary line and midclavicular line is the most common site of involvement. Mycobacterium tuberculosis and Actinomyces species are the most common pathogens. ${ }^{1}$ Other reported pathogens include Blastomycosis, Aspergillus, Nocardia, Mucormycosis, and Fusobactrium species. To our knowledge, only a few cases of empyema necessitatis due to Streptococcus pneumonia ${ }^{3}$ have been reported in adults and even fewer due to $S$. aureus infection. ${ }^{4}$ Chest wall infection could be from direct extension of pulmonary infection or 

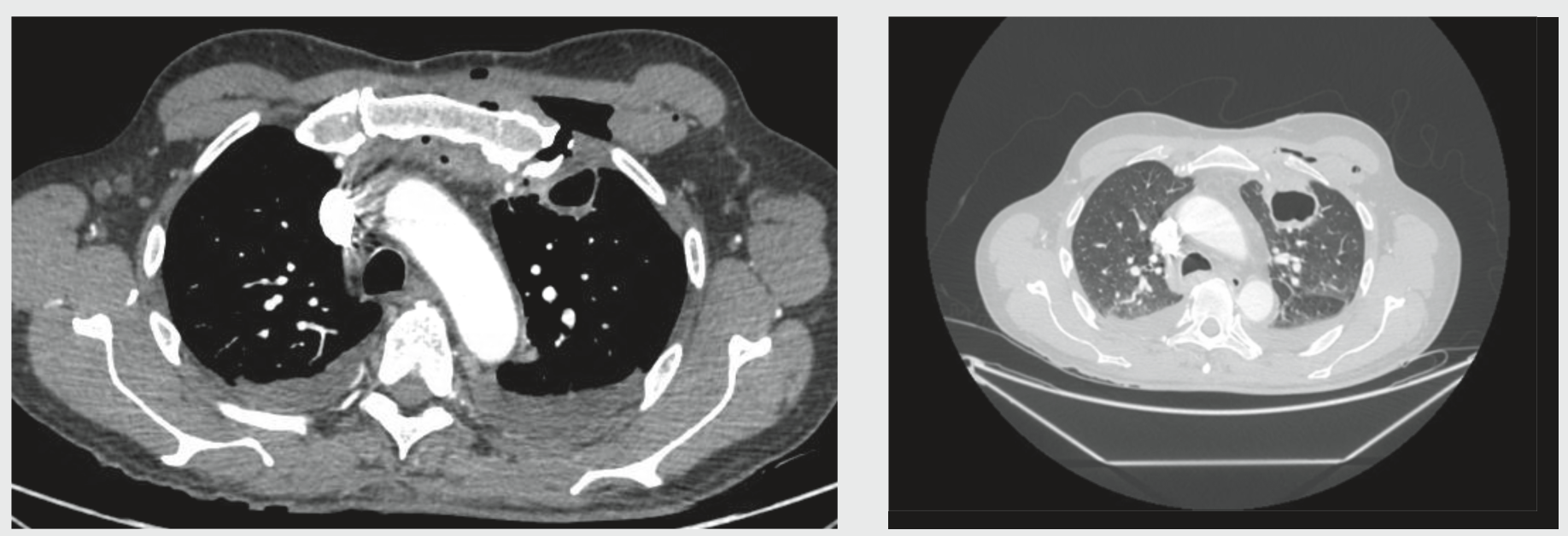

Figure 1. Gas locules extending from the LUL parenchymal cavity into the pleural space and the anterior chest wall, involving the left sternoclavicular joint, the pectoralis muscles, and the retrosternal space. Bilateral pleural effusions are noted.

from spread of the pathogens to the chest wall through the lymphatic system. ${ }^{2}$ Risk factors include immunocompromised states, including diabetes and alcoholism, and recurrent aspiration. Empyema necessitatis due to $S$. aureus has been reported to occur in patients with a history of chest surgery or trauma. ${ }^{4}$ This patient lives at home and has no recent medical or surgical problems. Additionally, for his hip pain, he underwent arthrocentesis and culture revealed MSSA; his sputum culture grew MRSA. This suggests that the lung abscess was unrelated to the hip septic arthritis.

Chest radiographs can be highly nonspecific or even normal. Chest CT is the best modality to examine the extent of infection. It typically demonstrates a collection (empyema) with extension into the chest wall. A chest wall mass/bulge filled with air and/or fluid communicating with the pleural space can be seen. The connections between the pleural and extra-pleural collections can be very small in size and occasionally are invisible. The lung parenchyma may show a cavity or infiltrates depending on the causative organism. Treatment consists of antimicrobials and drainage of the abscess usually with a surgical intervention.

Keywords: lung abscess, chest wall abscess, empyema, Staphylococcus aureus
From: The Department of Internal Medicine at Texas Tech University Health Sciences Center in Lubbock, TX.

Submitted: $6 / 26 / 2017$

Accepted: $7 / 10 / 2017$

Reviewer: Eman Attaya MD

Conflicts of interest: none

\section{REFERENCES}

1. Herrak L, Msouger Y, Ouadnouni Y, et al. Pulmonary actinomycosis with chest wall fistula formation. Rev Mal Respir 24: 349-352, 2007.

2. Koreeda Y, Hirotsu Y, Fukunaga H, et al. A case of tuberculous abscess in the chest wall close to the thickening pleural lesion following tuberculous pleuritis. Nihon Kyobu Shikkan Gakkai Zasshi 35: 1013-1019, 1997.

3. Ko Y, Tobino K, Yasuda Y, Sueyasu T, Nishizawa S. A community-acquired lung abscess attributable to Streptococcus pneumoniae which extended directly into the chest wall. Intern Med. 2017; 56(1):109-113.

4. Chaudhry LA, A Ba Mousa A, Zamzami M, Robert AA. Contemporary empyema thoracis necessitans in an adult male caused by Staphylococcus aureus: decortication is superior to traditional under water seal intercostal tube in chronic empyema. Pan Afr Med J. 2015; 20: 115. 2015 Feb 9. doi: 10.11604/pamj.2015.20.115.5865 